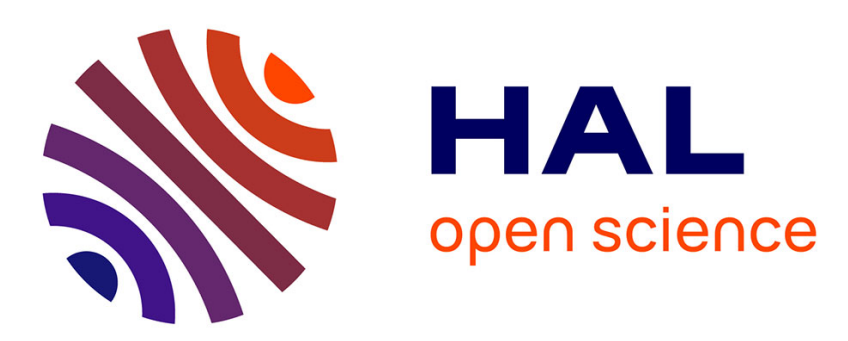

\title{
Efficient cluster-based routing algorithm for body sensor networks
}

Nadine Boudargham, Jacques Abdo, Jacques Demerjian, Christophe Guyeux, Talar Atechian

\section{> To cite this version:}

Nadine Boudargham, Jacques Abdo, Jacques Demerjian, Christophe Guyeux, Talar Atechian. Efficient cluster-based routing algorithm for body sensor networks. Middle East and North Africa COMMunications Conference, Apr 2018, Jounieh, Lebanon. hal-01992721

\section{HAL Id: hal-01992721 \\ https://hal.science/hal-01992721}

Submitted on 24 Jan 2019

HAL is a multi-disciplinary open access archive for the deposit and dissemination of scientific research documents, whether they are published or not. The documents may come from teaching and research institutions in France or abroad, or from public or private research centers.
L'archive ouverte pluridisciplinaire HAL, est destinée au dépôt et à la diffusion de documents scientifiques de niveau recherche, publiés ou non, émanant des établissements d'enseignement et de recherche français ou étrangers, des laboratoires publics ou privés. 


\section{Efficient Cluster-Based Routing Algorithm for Body Sensor Networks}

\author{
Nadine Boudargham \\ Faculty of Engineering \\ Notre Dame University \\ Deir El Kamar, Lebanon \\ nboudargham@ndu.edu.lb
}

\author{
Jacques Bou Abdo \\ Faculty of Natural and Applied Sciences \\ Notre Dame University \\ Deir El Kamar, Lebanon \\ jbouabdo@ndu.edu.lb
}

\author{
Jacques Demerjian \\ LARIFA-EDS, Faculty of Sciences \\ Lebanese University \\ Fanar, Lebanon \\ jacques.demerjian@ul.edu.lb
}

\author{
Christophe Guyeux \\ FEMTO-ST Institute, UMR 6174 CNRS \\ Université de Bourgogne \\ Franche-Comté Besançon, France \\ christophe.guyeux@univ-fcomte.fr
}

\author{
Talar Atechian \\ TICKET Lab, Faculty of Engineering \\ Antonine University \\ Baabda, Lebanon \\ talar.atechian@ua.edu.lb
}

\begin{abstract}
Body Sensor Networks have gained a lot of research interest lately for the variety of applications they can serve. In such networks where nodes might hold critical information about people's lives, designing efficient routing schemes is very important to guarantee data delivery with the lowest delay and energy consumption. Even though some cluster-based routing schemes were proposed in the literature, none of them offer a complete solution that guarantees energy and delay efficient routing in BSN. In this paper, we propose a robust clusterbased algorithm that increases the routing efficiency through every step of the routing process: cluster formation, cluster head election, and routing operation of data to the sink. The proposed algorithm is compared to existing cluster-based schemes, and MATLAB simulations are performed to assess the performance of the proposed algorithm with respect to delay and energy consumption.
\end{abstract}

Keywords-Body Sensor Networks (BSNs); Cluster-based Routing; Delay; Energy Consumption.

\section{INTRODUCTION}

Body Sensor Networks (BSNs) consist of smart wearable and implanted medical sensors able to collect physiological data from the human body and send them wirelessly through a sink node to health care providers for analysis and decision making [5]. Research interest in BSN field has remarkably increased during the past few years for its ability to enhance people's lives, and the variety of medical and non-medical applications it can serve such as anomaly identification, emotion detection, security authentication, entertainment, sports, etc. [6], [4].

In BSNs, nodes have very limited power, and their batteries cannot be changed frequently, specially when they are implanted inside the human body. Also, the sensed data can be very critical and requires fast actions to be taken to save the person's life. Therefore, there is a need to develop efficient and reliable routing schemes that guarantee data delivery with the lowest delay and energy consumption.
Cluster-based routing is proven to be very efficient in prolonging BSN lifetime through spreading the energy consumption equally among body nodes [17], [2]. In clusterbased routing, nodes are grouped into different clusters and one Cluster Head $(\mathrm{CH})$ is elected per cluster. Nodes inside the cluster send their data to the $\mathrm{CH}$, and only the $\mathrm{CH}$ can transmit the data to the sink. This will decrease the number of active nodes in the network leading to lower energy consumption and delay.

The remainder of the paper is organized as follows. Related work is presented in Section II. A new efficient cluster-based routing algorithm is proposed in Section III. Simulation and comparison of the proposed scheme to existing routing algorithms, as well as discussion of the corresponding delay and energy consumption performance, are presented in Section IV. This research work ends by a conclusion section, in which the proposal is summarized and future work is outlined.

\section{RELATED WORK}

Few cluster-based routing schemes for BSNs are discussed in the literature, each differs from the other by the way clusters are formed, the criteria used to elect the $\mathrm{CH}$ of each cluster, and the routing process for data to reach the sink.

One of the simplest cluster-based routing is Low-Energy Adaptive Clustering Hierarchy (LEACH) protocol proposed in [8]. In LEACH, nodes are selected as CHs by random rotation based on a certain probability computation, and the other nodes join the nearest $\mathrm{CH}$ to form a cluster. LEACH allows every node to become a $\mathrm{CH}$ in a round which distributes the energy consumption among all nodes of the network. However, since $\mathrm{CHs}$ are randomly elected, the other non- $\mathrm{CH}$ nodes might be located out of the communication range of the elected $\mathrm{CHs}$, and cannot therefore join a cluster; also, the number of nodes joining a cluster can be large, which would quickly exhaust the energy of the corresponding $\mathrm{CH}$. In addition, direct transmission of data from nodes to the $\mathrm{CH}$ and from the $\mathrm{CH}$ to the sink is used, which might not be efficient if the distance 
between the nodes and the $\mathrm{CH}$ or between the $\mathrm{CH}$ and the sink is large.

Another cluster-based scheme is the Improved LEACH protocol for WBAN presented in [20]. Improved LEACH is an enhancement over the LEACH protocol since $\mathrm{CH}$ are not only elected based on a certain probability, but also based on the residual energy of the nodes and the nodes type; i.e., less important nodes with the highest residual energy are chosen to be the $\mathrm{CHs}$ in a round. The problem with Improved LEACH is that even though it elects $\mathrm{CHs}$ based on significant parameters, it ignores other important criteria such as the nodes' distance to the sink, their mobility, and their communication range. Also, like in LEACH, the number of cluster members might be high in Improved LEACH, and direct transmission of data is adopted which decreases the routing efficiency.

Authors in [7] proposed the Hybrid Indirect Transmission (HIT) protocol. In HIT, CHs are elected by random rotation in a similar way as LEACH. However, unlike LEACH where cluster members send their data via direct transmission to the elected $\mathrm{CH}$, data is sent indirectly via multi-hop route from the nodes to the $\mathrm{CH}$ in order to further minimize the energy consumption of nodes. As LEACH, the problem with HIT arises from the random way the $\mathrm{CHs}$ are elected, ignoring many parameters important to be considered in BSNs.

In [18], authors suggest a cluster-based routing algorithm in which nodes are elected as $\mathrm{CHs}$ if both the ratio of their current energy to the average energy of the network and their selection probability computed based on Integer of Linear Programming (IPL) are high. All non- $\mathrm{CH}$ nodes located in the region between the elected $\mathrm{CH}$ and the sink will become cluster members. This algorithm also ignores many important parameters in the election of $\mathrm{CHs}$, and can also drain the energy of the $\mathrm{CH}$ quickly if the number of cluster members is high.

Authors in [19] propose the Anybody protocol, in which nodes with the highest density are elected as $\mathrm{CHs}$, where the density is computed as the ratio of the number of links to the number of nodes within two-hop neighborhood. The data is then sent from the cluster members to the elected $\mathrm{CH}$ via a multi-hop intra-cluster path, and from the $\mathrm{CH}$ to the sink through a multi-hop inter-cluster route. Since the $\mathrm{CH}$ election is based on highest density, this algorithm may not be efficient when the number of cluster members is high, specially that other important criteria such as energy of nodes, distance to the sink, etc. are not considered in the $\mathrm{CH}$ election process.

None of the previous research works discussed above offer a complete solution that guarantees energy and delay efficient routing in BSN. They either fail to consider important criteria to elect the best $\mathrm{CHs}$ that would lead to better routing efficiency, or they ignore the importance of limiting the number of nodes in a cluster to prevent fast drainage of $\mathrm{CH}$ energy, or they adopt inefficient routing operation of data like direct transmission.

We therefore propose a robust and efficient routing algorithm that decreases the energy consumption and delay through every step of the routing process: the clusters formation, the $\mathrm{CHs}$ election, and the routing operation of data to the sink. The proposed algorithm presents a complete solution that guarantees efficient routing of data which is critical in BSNs.

\section{Proposed Routing Algorithm}

As stated earlier, the proposed algorithm considers increasing the routing efficiency through each of the following steps of the routing process:

1) Clusters formation

2) $\mathrm{CH}$ election

3) Routing operation: Intra and Inter cluster routing.

\section{A. Energy Model}

The first order energy model widely adopted in BSN studies [16], [18], [13], [9] is used in the proposed scheme. The corresponding transmitter and receiver energy are as follows:

$$
\begin{gathered}
E_{T X}(L, d)=E_{T x-\text { elec }} \cdot L+\epsilon_{\text {amp }} \cdot L \cdot d^{n} \\
E_{R X}(L)=E_{R x-\text { elec }} \cdot L
\end{gathered}
$$

$E_{T x-e l e c}$ and $E_{R x-e l e c}$ represent the energy consumed by the electronic circuits of the transmitter and the receiver, and $\epsilon_{a m p}$ represents the energy consumed by the transmit amplifier [15]. These values depend on the type of the transceiver used. We consider using Nordic nRF24L01 2.4 $\mathrm{GHz}$ transceivers that are frequently used in BSNs [13], [16], [10]. $L$ represents the packet size, and $n$ indicates the average Path Loss (PL) exponent of the whole body, which is equal to 3.38 [14].

\section{B. Clusters Formation}

To guarantee efficient routing, the proposed algorithm calculates the optimal number of clusters using Equation (3). This formula computed in [3], [11] is chosen since it evaluates the best number of clusters that minimizes the total energy consumption in the network which is our main concern.

$$
k_{\text {optimal }}=\sqrt{\frac{N_{s} \cdot \epsilon_{f s} \cdot A}{2 \pi \cdot \epsilon_{\text {amp }} \cdot d_{\text {toSink }}^{n}}}
$$

where:

$k_{\text {optimal }}$ : Optimal number of cluster.

$N_{s}$ : Number of nodes distributed on and in the body.

$\epsilon_{f s}$ : Energy of amplifier in free space obtained by com-

puting $\epsilon_{a m p}$ in equation (1) for $\mathrm{n}=2$.

$A:$ MxM sensing area.

$\epsilon_{a m p}$ : Energy of amplifier in multi-path fading.

$d_{\text {toSink }}$ : Average distance from transmitting nodes to the sink. $n$ : Average PL exponent of the whole body.

$k_{\text {optimal }}$ clusters will then be formed, each of which encloses $N_{c}$ nodes, where: 
TABLE I: PL EXPONENT FOR DIFFERENT BODY PARTS

$$
N_{c}=\frac{N_{s}}{k_{\text {optimal }}}
$$

The above formula guarantees that nodes are fairly distributed between clusters, which reduces the energy consumption in the network since the presence of large number of nodes in a cluster will quickly drain the energy of the elected $\mathrm{CH}$.

Also, in order to reduce the computation overhead, recluster formation only occurs when nodes move outside their assigned cluster. Re-clustering computation is therefore avoided as long as the nodes remain in their assigned clusters even when their location changes due to motion.

\section{C. $\mathrm{CH}$ Election}

Electing the appropriate $\mathrm{CH}$ of every cluster is important to guarantee efficient routing. For instance, choosing improper $\mathrm{CH}$ can increase the delay and energy consumption of the network due to transmission of data over longer distances, and re-transmission of failed packets to the sink. For this reason, the proposed algorithm considers the following parameters to elect the best $\mathrm{CHs}$ that would lead to better routing efficiency:

- Distance between the nodes and the sink;

- Residual energy of nodes;

- Transmission Scope (TS);

- Mobility of nodes;

- $\quad$ Node index.

For every node, the probability to become a $\mathrm{CH}$ is computed based on the following formula:

$$
P_{x}=\frac{E_{x} \cdot T S_{x} \cdot I}{d_{\text {toSink }} \cdot M_{x}}
$$

where:

$P_{x}$ : Probability for node $\mathrm{x}$ to become a $\mathrm{CH}$.

$E_{x}$ : Residual energy of node $\mathrm{x}$.

$T S_{x}$ : Transmission Scope of node $\mathrm{x}$.

$I$ : Node Index.

$d_{\text {toSink }}$ : Distance from the transmitting node to the sink.

$M_{x}$ : Mobility factor of node $\mathrm{x}$.

Equation (5) suggests that in every cluster, the node with the highest energy, TS, and index value, along with the lowest mobility and the shortest distance to the sink, will have the highest probability to be elected as $\mathrm{CH}$.

1) Transmission Scope Computation: The Transmission Scope (TS) of every node is defined as:

$$
T S_{x}=\frac{1}{\text { PL exponent of node } \mathrm{x}}
$$

\begin{tabular}{||c|c||}
\hline Body Part & PL Exponent (n) \\
\hline \hline Arm & 3.35 \\
\hline Leg & 3.45 \\
\hline Torso & 3.23 \\
\hline Back & 2.18 \\
\hline Implented and Non-LOS Nodes & 5.9 \\
\hline
\end{tabular}

TS reveals the connectivity and coverage strength of a node. It is computed as the inverse of the PL exponent of every node since the PL parameter depends on the environment type and the medium of propagation, and includes all types of losses in the network: reflection, refraction, absorption, and diffraction [1]. Equation (6) implies that the lower is the PL exponent of a node, the higher is its TS value.

Since nodes are placed on different parts of the body, their PL exponent value is not similar. It ranges between 2.18 and 5.9 depending on the node's location as represented in Table I [14].

2) Mobility Factor Computation: Not all BSN nodes are stationary. For instance, nodes located on/in the arms and legs are mobile, and therefore, it's important to consider nodes' mobility in the election of the $\mathrm{CH}$. [12]:

The mobility factor of every node is computed as follows

$$
M_{i}(t)=\frac{1}{N_{c}-1} \sum_{j=0}^{N_{c}-1} d_{i j}(t)
$$

where $d_{i j}$ is the distance between the node $i$ to other node $j$ in the same cluster, and $N_{c}$ represents the number of nodes in a cluster.

The mobility factor in (7) calculates the sum of distances from every node in a cluster to the rest of the nodes in this cluster. If the node moves away from the others, the sum of distances will increase leading to a higher mobility factor.

3) Node Index: The proposed algorithm distinguishes between two types of nodes:

- Holders of important data: nodes capturing vital data like ECG, EEG and EMG, and nodes capturing critical data depending on the patient status. For instance, if the patient has a history with increased blood pressure, the blood pressure sensor is classified as a holder of important data. For this type of nodes, additional measures should be taken to preserve their energy; they are therefore assigned a low index value to decrease their probability to become $\mathrm{CHs}$.

- Holders of less important data: this type of nodes includes all the other sensors. They are assigned a higher index value to increase their probability to become CHs.

By taking into consideration the three parameters discussed above along with the residual energy of nodes and their 
distance to the sink, the most appropriate node in every cluster will be elected as $\mathrm{CH}$, increasing therefore the routing efficiency.

\section{Routing Operation}

The routing operation covers both intra- and inter-cluster routing. Intra-cluster routing is the process of delivering data from the cluster members to the corresponding $\mathrm{CH}$, i.e. the process of data transmission inside every cluster. And intercluster routing refers to the process of transmitting data from the different $\mathrm{CHs}$ in the network to the final destination (the $\operatorname{sink}$ ).

In the proposed algorithm, multi-hop route is used for both intra- and inter-cluster routing since it reduces the energy consumption of nodes compared to direct routing schemes [7]. For intra-cluster routing, a Forwarding Cost (FC) is computed for every node inside a cluster to select the best forwarder using the following formula:

$$
F C_{x}=\frac{d_{t o C H}}{E_{x} \cdot T S_{x}}
$$

where:

$d_{t o C H}$ : Distance between node $\mathrm{x}$ belonging to a cluster and the elected $\mathrm{CH}$ of that cluster.

$E_{x}$ : Residual energy of node $\mathrm{x}$.

$T S_{x}$ : Transmission Scope of node $\mathrm{x}$ depending on its location.

The node with the lowest FC (i.e., with the shortest distance to the cluster elected $\mathrm{CH}$, and with the highest residual energy and TS) is selected as a forwarder, and neighboring nodes send their data to this elected node.

Similarly, in inter-cluster routing, the $\mathrm{FC}$ of every $\mathrm{CH}$ is computed, and the $\mathrm{CH}$ with the lowest $\mathrm{FC}$ is selected as the forwarder of data to the sink:

$$
F C_{C H}=\frac{d_{t o S i n k}}{E_{C H} \cdot T S_{C H}}
$$

The flow chart of the proposed algorithm is presented in Fig. 1. It illustrates the three steps of the suggested scheme: cluster formation, $\mathrm{CH}$ election, and routing process (intra- and inter-cluster routing). The flow chart shows that the proposed algorithm presents a complete solution that guarantees efficient routing through dividing the BSN into optimal number of clusters, considering all the important parameters in the election of $\mathrm{CHs}$, and adopting a multi-path route of data to the destination.

\section{Simulation of the Proposed Scheme}

In this section, the proposed algorithm is compared to existing cluster-based routing schemes. Delay and energy consumption simulations are performed and the obtained results are discussed to assess the performance of the proposed scheme with respect to the others.

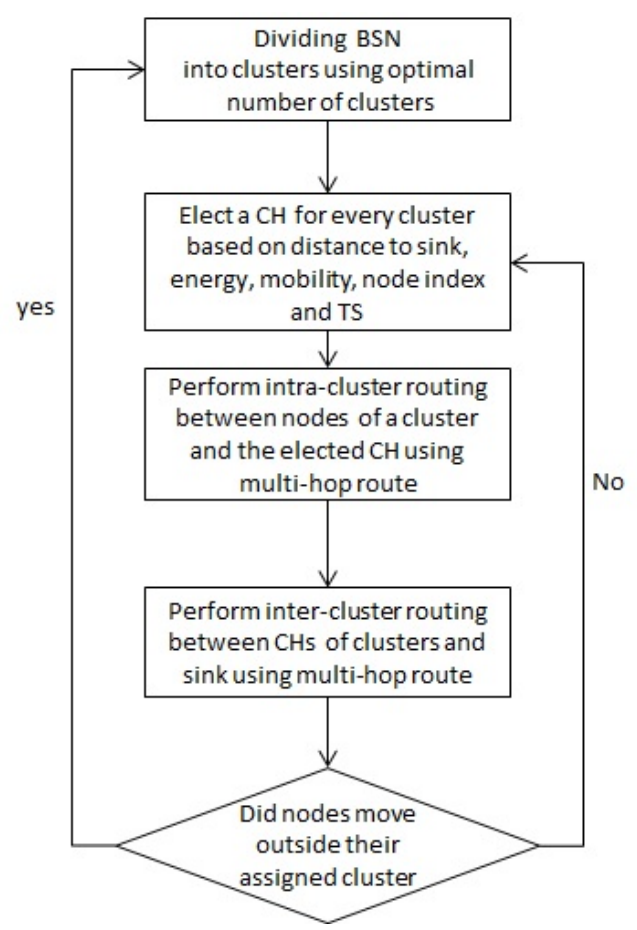

Fig. 1: Proposed Algorithm Flow Chart

\section{A. Simulation Parameters}

In order to assess the performance of the proposed algorithm, we consider a BSN formed of 25 sensors distributed all around the human body as per Fig. 2. 20 sensors are placed on the body (wearable sensors) and 5 sensors are either implanted or placed on the back (Non-LOS nodes). To study the effect of mobility, we consider that the arms can move from left to right and vice versa; and therefore the 6 nodes placed on/in the arms are considered to be mobile. We also consider that around $25 \%$ of the nodes (6 out of 25) are holders of important data and are therefore assigned low index value.

The simulation parameters are summarized in Table II.

The proposed algorithm is compared to LEACH and Improved LEACH cluster-based routing schemes with respect to delay and energy consumption. Simulations were performed using MATLAB R2014b.

\section{B. Results and Discussion}

The cumulative delay induced by the proposed algorithm, $\mathrm{LEACH}$, and Improved LEACH is presented in Fig. 3. Results show that the proposed algorithm outperforms the other schemes, since it takes into account the mobility, the node index, and the transmission scope of the nodes, in addition to the nodes' energy and distance to the sink to elect the $\mathrm{CHs}$. The other algorithms either elect the $\mathrm{CHs}$ randomly by rotation $(\mathrm{LEACH})$, or only consider the residual energy of nodes and their level of importance (Improved LEACH) without accounting for the medium condition surrounding the nodes, nor their distance to the sink, nor their mobility. The election of unsuitable $\mathrm{CHs}$ leads to higher delays induced by transmission of data over longer distances or through too many obstacles, or by re-transmission of the failed packets to 


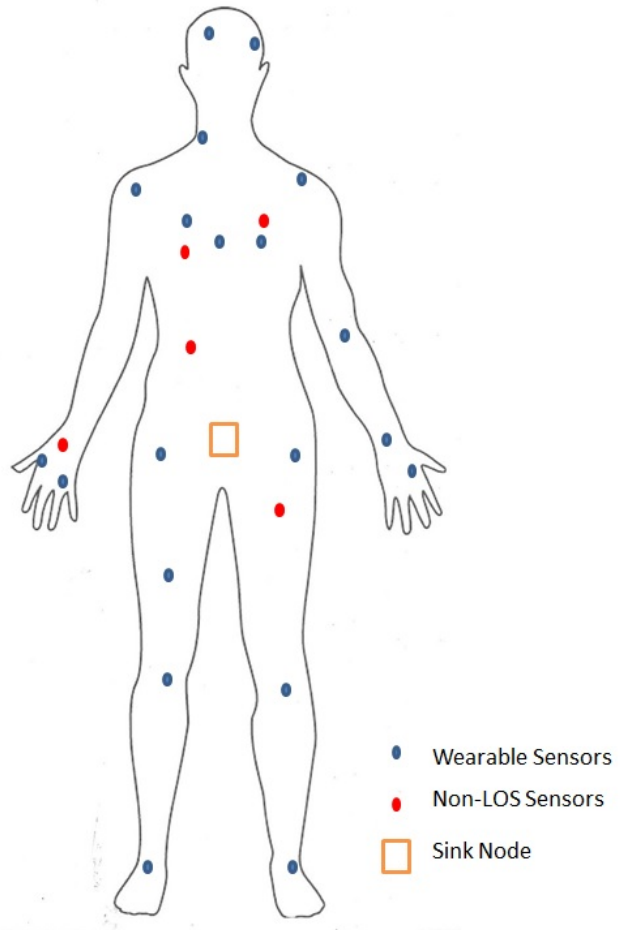

Fig. 2: Nodes Distribution

the sink. Also, optimal number of clusters is formed in the proposed scheme, which further decreases the total delay in the network.

Fig. 4 illustrates the energy consumed by the nodes in different rounds for the three compared schemes. It shows that the proposed scheme performs better than the other algorithms. For instance, the LEACH protocol consumes the highest energy since $\mathrm{CHs}$ are elected randomly, thus much more energy is needed to transmit data to the sink when the distance between $\mathrm{CHs}$ and sink is large, or when the transmission scope of the $\mathrm{CH}$ is low, or when $\mathrm{CHs}$ move away from the cluster nodes.

TABLE II: SIMULATION PARAMETERS

\begin{tabular}{||l|c||}
\hline Simulation Parameters & Value \\
\hline \hline Distribution Area & $2 \mathrm{~m} \times 2 \mathrm{~m}$ \\
\hline Sink Location & Center of Body \\
\hline Number of Nodes $\left(N_{s}\right)$ & 25 \\
\hline Number of wearable Nodes & 20 \\
\hline Number of Implanted or Non-LOS Nodes & 5 \\
\hline Number of moving Nodes & 6 \\
\hline Number of Holders of Important Data & 6 \\
\hline$E_{T x-\text { elec }}$ & $16.7 \mathrm{~nJ} / \mathrm{bit}$ \\
\hline$E_{R x-\text { elec }}$ & $36.1 \mathrm{~nJ} / \mathrm{bit}$ \\
\hline$\epsilon_{a m p}$ & $1.97 \mathrm{~nJ} / \mathrm{bit}$ \\
\hline$\epsilon_{f s}$ & $10.9 \mathrm{~nJ} / \mathrm{bit}$ \\
\hline PL Exponent of Nodes & $2.18-5.9$ \\
\hline PL exponent of Whole Body $(\mathrm{n})$ & 3.38 \\
\hline Packet Size $(\mathrm{L})$ & $4000 \mathrm{bits}$ \\
\hline Clusters Density $N_{c}$ & $N_{s} / K_{o p t}$ \\
\hline
\end{tabular}

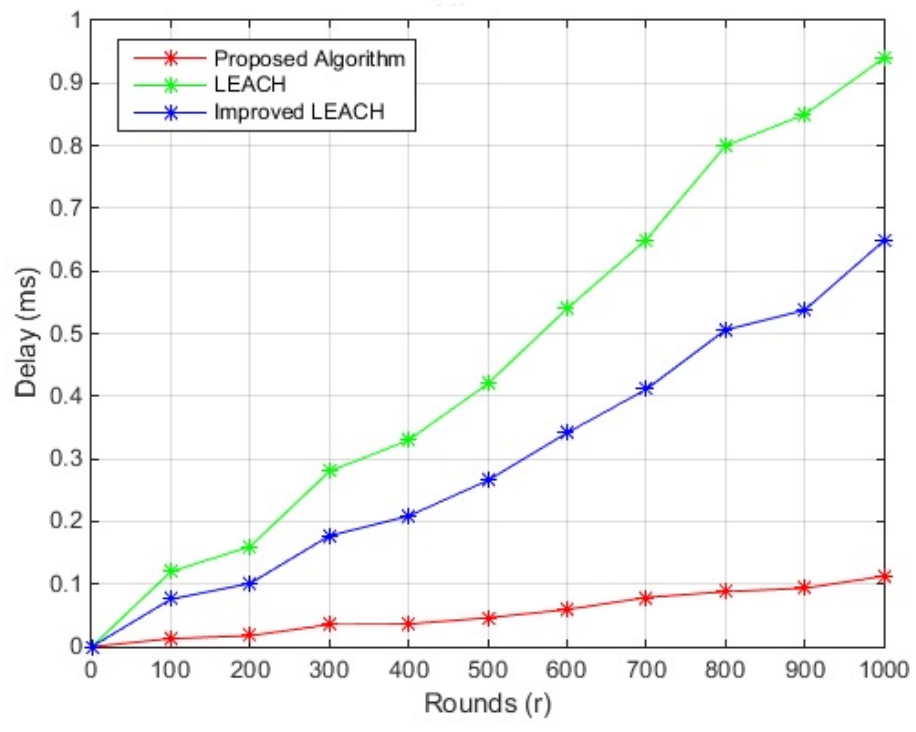

Fig. 3: Delay Performance of Different Routing Schemes

The energy consumption of the Improved LEACH is high since it does not take the distance to the sink, the mobility, and the transmission scope of the nodes into consideration. However, it performs better than LEACH protocol, since it accounts for the energy of nodes and their level of importance in the $\mathrm{CH}$ election, which would better distribute the energy consumption between nodes. Also, both LEACH and Improved LEACH used direct transmission of data from cluster members to $\mathrm{CHs}$, and from $\mathrm{CHs}$ to the sink, whereas the proposed algorithm follows a multi-hop intra- and inter-cluster routing which further decreases the nodes' energy consumption. This is in addition to the fact that the proposed algorithm divides the BSN into optimal number of clusters to provides minimum energy consumption, whereas the other two schemes elect the $\mathrm{CHs}$ first, and clusters will then be formed by allowing non$\mathrm{CH}$ members to join the closest $\mathrm{CH}$, without accounting for

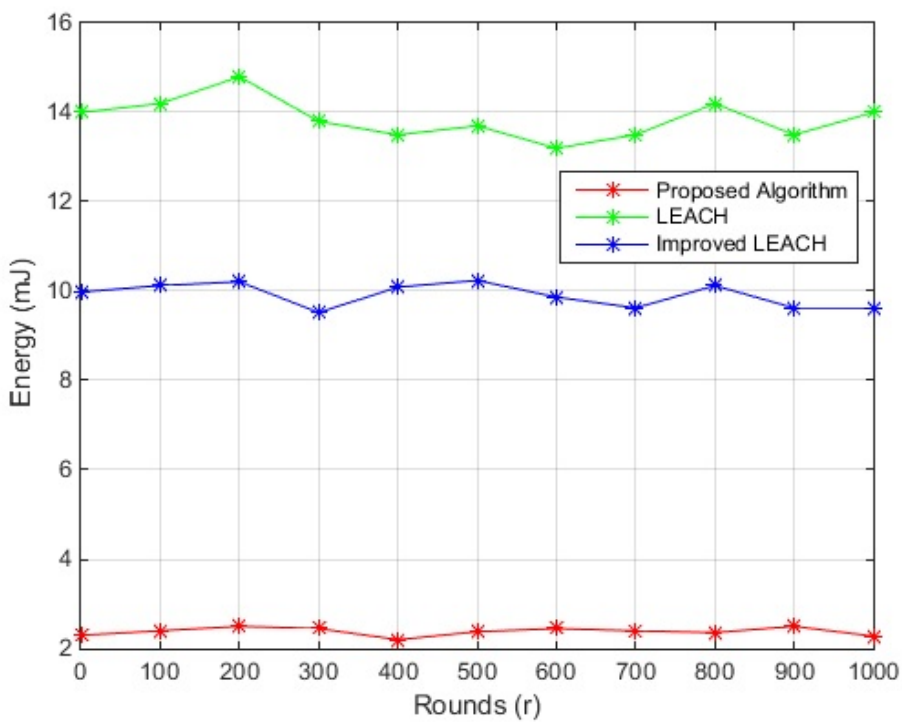

Fig. 4: Energy Performance of Different Routing Schemes 
the cluster size nor the number of formed clusters.

The results prove that the proposed algorithm succeeds in providing a reliable and efficient routing in BSN through decreasing the delay and energy consumption of every step of the routing process.

\section{CONCLUSION AND Future WORK}

This paper proposed a robust and efficient cluster-based routing algorithm that works on decreasing the delay and energy consumption through the three steps of the routing process: cluster formation, $\mathrm{CH}$ election, and routing operation of data to the sink. The proposed scheme was compared to other existing cluster-based algorithms with respect to delay and energy consumption. Results showed that it outperforms the others since it provides a solution that guarantees efficient data delivery. The proposed algorithm is therefore highly suitable for BSN applications. Future work includes investigating Medium Access Control (MAC) protocols that guarantee energy efficiency in BSNs as well as exploring security mechanisms for this type of networks.

\section{ACKNOWLEDGMENT}

This work is partially funded with support from the Lebanese University Research Program (Number: 4/6132), the Labex ACTION program (contract ANR-11LABX-01-01), and the France-Suisse Interreg RESponSE project.

\section{REFERENCES}

[1] Vital Connect Website, URL: https://www.utdallas.edu/ torlak/courses/ee4367/lectures/lectureradio.pdf.

[2] T. A. Alghamdi. Cluster based energy efficient routing protocol for wireless body area networks. Trends in Applied Sciences Research, 11(1):12, 2016

[3] N. Amini, A. Vahdatpour, W. Xu, M. Gerla, and M. Sarrafzadeh. Cluster size optimization in sensor networks with decentralized cluster-based protocols. Computer Communications, 35(2):207-220, 2012.

[4] J. M. Bahi, C. Guyeux, A. Makhoul, and C. Pham. Low cost monitoring and intruders detection using wireless video sensor networks. International Journal of Distributed Sensor Networks, 2012, 2012.

[5] N. Boudargham, J. Abdo, J. Demerjian, C. Guyeux, and A. Makhoul. Investigating low level protocols for wireless body sensor networks. 3rd ACS/IEEE International Conference on Computer Systems and Applications, November 2016.

[6] N. Boudargham, J. Bou Abdo, J. Demerjian, and C. Guyeux. Exhaustive study on medical sensors. 11th SENSORCOMM International Conference on Sensor Technologies and Applications, September 2017.

[7] B. J. Culpepper, L. Dung, and M. Moh. Design and analysis of hybrid indirect transmissions (hit) for data gathering in wireless micro sensor networks. ACM SIGMOBILE Mobile Computing and Communications Review, 8(1):61-83, 2004.

[8] W. R. Heinzelman, A. Chandrakasan, and H. Balakrishnan. Energyefficient communication protocol for wireless microsensor networks. 33rd Hawaii International Conference on System Sciences, pages 10 pp, 2000.

[9] N. Javaid, Z. Abbas, M. Fareed, Z. Khan, and N. Alrajeh. M-attempt: A new energy-efficient routing protocol for wireless body area sensor networks. Procedia Computer Science, 19:224-231, 2013.

[10] N. Javaid, A. Ahmad, Q. Nadeem, M. Imran, and N. Haider. imsimple: improved stable increased-throughput multi-hop link efficient routing protocol for wireless body area networks. Computers in Human Behavior, 51:1003-1011, 2015.

[11] V. Kumar. Cluster size optimization in gaussian distributed wireless sensor networks. International Journal of Engineering and Technology (IJET), 6:1581-1592, 012014.
[12] P. Maurya and A. Kaur. A survey on descendants of leach protocol International Journal of Information Engineering and Electronic Business, 8(2):46, 2016.

[13] Q. Nadeem, N. Javaid, S. N. Mohammad, M. Khan, S. Sarfraz, and M. Gull. Simple: Stable increased-throughput multi-hop protocol for link efficiency in wireless body area networks. 8th International Conference on Broadband and Wireless Computing, Communication and Applications (BWCCA), pages 221-226, 2013.

[14] E. Reusens, W. Joseph, B. Latré, B. Braem, G. Vermeeren, E. Tanghe, L. Martens, I. Moerman, and C. Blondia. Characterization of onbody communication channel and energy efficient topology design for wireless body area networks. IEEE Transactions on Information Technology in Biomedicine, 13(6):933-945, 2009.

[15] M. M. Sahndhu, N. Javaid, M. Imran, M. Guizani, Z. A. Khan, and U. Qasim. Bec: A novel routing protocol for balanced energy consumption in wireless body area networks. International Wireless Communications and Mobile Computing Conference (IWCMC), 2015.

[16] A. Tauqir, N. Javaid, S. Akram, A. Rao, and S. N. Mohammad. Distance aware relaying energy-efficient: Dare to monitor patients in multi-hop body area sensor networks. 8th Conference on Broadband and Wireless Computing, Communication and Applications (BWCCA), pages 206213, 2013.

[17] M. T. I. ul Huque, K. S. Munasinghe, M. Abolhasan, and A. Jamalipour. Sea-ban: Semi-autonomous adaptive routing in wireless body area networks. 7th International Conference on Signal Processing and Communication Systems (ICSPCS), pages 1-7, 2013.

[18] M. Verma and R. Rai. Energy-efficient cluster-based mechanism for wban communications for healthcare applications. International Journal of Computer Applications, 120(19), 2015.

[19] T. Watteyne, I. Augé-Blum, M. Dohler, and D. Barthel. Anybody: a selforganization protocol for body area networks. 2nd ICST International Conference on Body Area Networks, page 6, 2007.

[20] J.-J. Zhang, X. Feng, Y.-L. Wang, T. Li, and W.-L. Yang. Research on the wban structure based on leach protocol. International Conference on Advances in Mechanical Engineering and Industrial Informatics, 2015. 